\title{
Estado nutricional antropométrico, bioquímico e ingesta alimentaria en niños escolares de 6 a 14 años, General Pueyrredón, Buenos Aires, Argentina Anthropometric and biochemical assessment of nutritional
status and dietary intake in school children aged 6-14 years,
Province of Buenos Aires, Argentina
}

\author{
Lic. Lorena Lázaro Cuesta, ${ }^{a}$ Dra. Analía Rearte, ${ }^{a}$ Dr. Sergio Rodríguez, \\ Lic. Melina Niglia, ${ }^{a}$ Lic. Horacio Scipioni, ${ }^{a}$ Bioq. Diego Rodríguez, ${ }^{a}$ \\ Lic. Rosalía Salinas, ${ }^{a}$ Sra. Claudia Sosa ${ }^{a}$ y Dra. Stella Rasse. ${ }^{a}$
}

\section{RESUMEN}

Introducción: La obesidad infantil es una epidemia global.Laedad escolary la adolescencia son etapas cruciales para la implementación de hábitos alimentarios y estilo de vida.

Objetivo: Evaluar el estado nutricional, antropométrico, bioquímicoeingesta alimentaria deniños, su relación con factores socioeconómicos y georreferenciación.

Métodos: Estudio transversal en escolares de 6 a 14 años, del Partido de General Pueyrredón, durante agosto-noviembre de 2013. Se evaluó la ingesta por recordatorio de 24 horas; se georreferenció con el programa gvSIG.

Resultados: Se incluyeron 1296 niños para evaluación antropométrica y socioeconómica. Una sub muestra incluyó 362 niños para parámetros bioquímicos e ingesta. El 42,97\% (IC 95\%: 40,3-45,7) presentó exceso de peso y el 18,5\% (IC 95\%: 16,3-20,5), obesidad. Desayunar se relacionó con menor riesgo de exceso de peso (OR: 0,7; IC 95\%: 0,5-0,9) y obesidad (OR: 0,7; IC 95\%:0,5-0,9). Concurrir al secundario se asoció a menor prevalencia de exceso de peso (OR:0,45; IC95\%:0,3-0,7); el sexomasculino presentómayor riesgo de obesidad (OR: 1,7; IC 95\%: 1,3-2,3). Presentaron anemia $4,44 \%$, hipercolesterolemia $19,6 \%$ ehipertrigliceridemia $21,3 \%$. La ingesta de lípidos y grasas saturadas fue alta y de colesterol dietario y fibra, baja. La distribución geográfica fue homogénea.

Conclusiones: La prevalencia de exceso de peso fue elevada. El riesgo de obesidad fue mayor en varones; el desayuno surgió como factor protector para sobrepeso/obesidad. La baja ingesta de fibras y elevada de grasas, y los niveles altos de colesterol y triglicéridos en sangre, reflejan que la malnutrición por exceso es un problema de salud pública prevalente.

Palabras clave: estado nutricional, obesidad, sobrepeso, nutrición del niño, nutrición del adolescente.

http:/ / dx.doi.org/10.5546/aap.2018.e34

Texto completo en inglés:

http:/ / dx.doi.org/10.5546/ aap.2018.eng.e34

Cómo citar: Lázaro Cuesta L, Rearte A, Rodríguez S, et al. Estado nutricional antropométrico, bioquímico e ingesta alimentaria en niños escolares de 6 a 14 años, General Pueyrredón, Buenos Aires, Argentina. Arch Argent Pediatr 2018;116(1):e34-e46.

\section{INTRODUCCIÓN}

En las últimas tres décadas, la obesidad infantil se ha convertido en una epidemia global. ${ }^{1-3}$ La edad escolar y la adolescencia son etapas cruciales para la configuración de los hábitos alimentarios y estilo de vida que persistirán en etapas posteriores, con repercusiones en la edad adulta e, incluso, en la senectud. ${ }^{4}$

La deficiencia de vitamina A constituye un problema de salud mundial, especialmente, en países en desarrollo. El hierro y el zinc son nutrientes preponderantes para el crecimiento y desarrollo adecuado. ${ }^{5}$ Las formas más frecuentes de malnutrición en población infantil son las carencias específicas de micronutrientes, que no pueden diagnosticarse a partir de antropometría. ${ }^{6}$ Estas deficiencias afectan el aprendizaje, crecimiento y desarrollo del niño. ${ }^{5}$

En la actualidad, América Latina se encuentra en una etapa de transición nutricional, fenómeno por el cual coexisten tanto la desnutrición como el sobrepeso, la obesidad y las carencias de micronutrientes. ${ }^{7}$ En Argentina, los últimos estudios han demostrado que, al menos, 3/10 niños o adolescentes tienen exceso de peso, ${ }^{8-10}$ con un aumento sostenido en los últimos años, en coincidencia con el resto del mundo. ${ }^{11}$ La mayoría de los estudios sobre estado nutricional están focalizados en niños preescolares y adolescentes, por lo que nuestro objetivo fue evaluar el estado nutricional antropométrico, 
bioquímico e ingesta alimentaria en niños escolarizados de 6 a 14 años del Partido de General Pueyrredón (PGP), su relación con factores socioeconómicos y georreferenciar los niños según el domicilio.

\section{POBLACIÓN Y MÉTODOS}

Estudio descriptivo, analítico, transversal entre agosto y noviembre de 2013 en el PGP (provincia de Buenos Aires -PBA-), que tenía 618989 habitantes, 81911 de 6-14 años (13,23\%). ${ }^{12}$ En 2013, el PGP contó con 323 unidades educativas. La tasa de escolaridad fue 94\% en el año 2006.

Se incluyeron, en la muestra, escolares de 6-14 años. Se solicitaron 12 horas de ayuno a los alumnos seleccionados para la submuestra. Se excluyeron niños con enfermedades crónicas e inmunosupresión primaria o secundaria.

El tamaño muestral calculado para la evaluación antropométrica fue de 1197 alumnos, considerando el evento menos prevalente (emaciación: $1,4 \%),{ }^{8}$ con una confianza del $95 \%$. Se dividió el PGP en tres zonas; se agruparon las ocho divisiones del consejo escolar; se seleccionaron aleatoriamente 22 escuelas y se respetó el porcentaje de públicas y privadas (60\% / 40\%, respectivamente) y la proporción de cada grupo etario. El muestreo de los grados también fue aleatorio.

Se tomó una submuestra para la evaluación de parámetros bioquímicos e ingesta alimentaria. El tamaño calculado fue de 300 niños, considerando una prevalencia del evento del 25\% (prevalencia de anemia), con una confianza del $95 \% .{ }^{13}$ De cada grado, se eligieron, al azar, de 5 a 6 niños para esta determinación.

Se recolectaron datos socioeconómicos mediante un cuestionario ad hoc, que fue completado por los padres, supervisados por personal entrenado (ver Anexo 1).

Los datos antropométricos se recolectaron en cada institución, por personal capacitado (ver Anexo 2). Para la medición de peso, se utilizaron balanzas portátiles marca CAM, código P-1001-PCADOBLE. Para la talla, cinta métrica inextensible sujeta sobre un plano vertical y una superficie rígida como plano horizontal. Las técnicas utilizadas se basaron en las guías de crecimiento y desarrollo de la Sociedad Argentina de Pediatría. ${ }^{14}$ La medición de la cintura se realizó en el borde superior de la cresta ilíaca con la "técnica de manos cruzadas", empleando una cinta metálica tipo Lufkin. Se utilizó la referencia de J. R. Fernández y col., con punto de corte en el percentilo $90 .{ }^{15}$ Se tomaron 2 mediciones y se calculó el promedio entre ellas.

Las muestras de laboratorio fueron tomadas por enfermeros entrenados y procesadas dentro de las 3 horas de su extracción. Se utilizaron tubos con ácido etilendiaminotetraacético (EDTA) (hemoglobina y hematocrito) y un contenedor sin anticoagulante para el resto de los analitos.

Las muestras fueron analizadas en el laboratorio del Hospital Interzonal Especializado Materno Infantil (HIEMI). Vitamina A, zinc y folato, en el laboratorio del Instituto de Desarrollo e Investigaciones Pediátricas "Prof. F. E. Viteri", Hospital de Niños "Sor María Ludovica" de La Plata.

Para datos de la ingesta, se capacitaron 11 nutricionistas con metodología estandarizada, que emplearon un recordatorio de $24 \mathrm{~h}$ de un día, modelos visuales y cartillas de alimentos. ${ }^{16,17}$ Se contabilizó la proporción de días de semana y fines de semana o feriados para evaluar la ingesta.

Se contó con dos equipos de 10 personas (enfermeros, nutricionistas, médicos, promotoras y asistentes sociales). Se completaron 2 escuelas por semana, con 3 visitas semanales a cada una. La devolución se realizó a cada alumno. Aquellos con alteraciones nutricionales se contactaron para la derivación a Pediatría.

Se incluyeron variables sociodemográficas (sexo, edad, nivel educativo de la madre, cobertura de salud, tipo de escuela privada/ pública, nivel de escolaridad primario/ secundario, cantidad de comidas diarias, hábito de desayunar, almorzar y cenar: $\geq 4$ veces por semana).

Variables antropométricas: clasificación y patrón de crecimiento infantil de la Organización Mundial de la Salud (OMS). ${ }^{18}$

El estado nutricional (índice de masa corporal -IMC- $)^{15,19}$ se clasificó en muy bajo peso: $\leq-3$ desvíos estándar (DE); bajo: $\leq-2 \mathrm{DE} />-3 \mathrm{DE}$; adecuado: $>-2 \mathrm{DE} /<+1 \mathrm{DE}$; sobrepeso: $\geq+1 \mathrm{DE} /<+2 \mathrm{DE}$; obesidad: $\geq+2 \mathrm{DE} /<+3 \mathrm{DE}$; obesidad grave: $\geq+3 \mathrm{DE}$. Se consideró exceso de peso a niños con sobrepeso, obesidad y obesidad grave. La circunferencia de cintura (CC) se consideró normal: < percentilo 90; y aumentada: $\geq$ percentilo 90 .

Se midieron variables bioquímicas: hematocrito y hemoglobina (bajo: < -2 DE de la media para edad y sexo como indicador de anemia), perfil lipídico, prealbúmina (nefelometría), ferritina (quimioluminiscencia), vitamina $\mathrm{A}$, zinc (espectrofotometría de absorción 

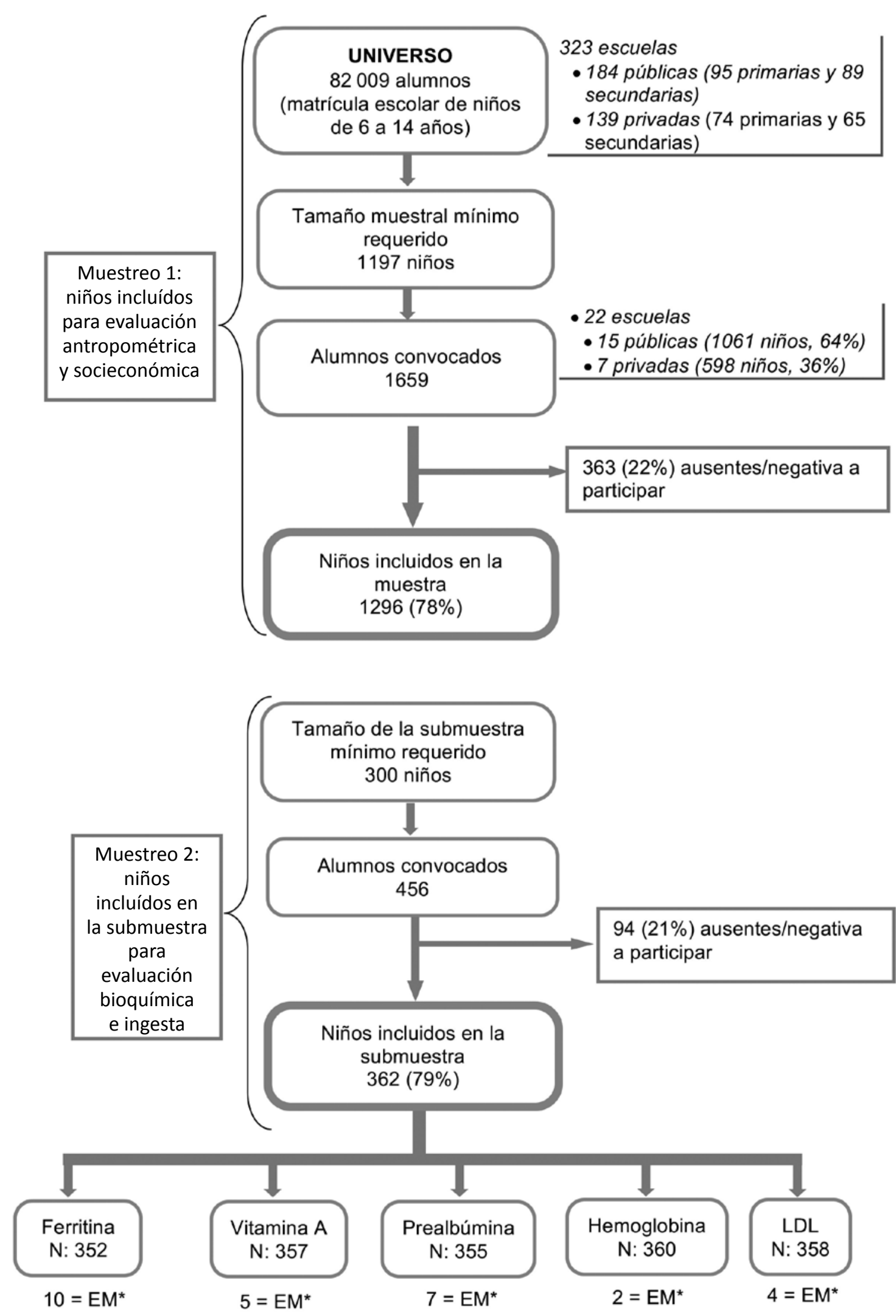

* EM: escasa muestra. 
atómica por llama, 213,9 nm) y folato.

Se evaluó la ingesta de calorías, proteínas, grasas, colesterol, hidratos de carbono, fibra, vitamina A, calcio, hierro, zinc y folatos.

La ingesta de grasas trans se calculó por diferencia respecto de las grasas totales como porcentaje respecto de la mediana de la energía consumida.

Consideraciones éticas: el estudio fue aprobado por el Comité de Bioética del HIEMI y la Comisión Conjunta de Investigación en Salud del Ministerio de Salud de la PBA.

Se solicitó el consentimiento informado firmado por padre, madre o tutor y el asentimiento a los alumnos.

\section{Análisis estadístico}

Para antropometría, se empleó el programa AnthroPlus (OMS); para la ingesta, el programa SARA (sistema de análisis y registro de alimentos) y, para análisis estadístico, Epi Info 7.

Se calculó mediana y rango intercuartílicos o media y DE para variables continuas y proporciones para datos categóricos con intervalos de confianza del 95\% (IC 95\%). Para datos continuos, se utilizó $t$ test o Wilcoxon rank sum y $\chi^{2}$ para proporciones. Se realizó un análisis multivariado mediante el modelo de regresión logística múltiple y se evaluaron variables asociadas a presentar exceso de peso y obesidad. Se utilizó como medida de asociación el odds ratio (OR) con IC 95\%. Se aceptó un error alfa del 5\%.

Para la ingesta, se calculó la mediana de nutrientes, de ingesta calórica y del porcentaje de macronutrientes respecto del valor calórico total (VCT). Se compararon los resultados con la ingesta dietaria de referencia (IDR). ${ }^{20}$

Para georreferenciar, se utilizó el programa gvSIG, para el manejo de información geográfica con precisión cartográfica, que permitió visualizar la distribución de la muestra. No se aplicaron test estadísticos.

\section{RESULTADOS}

Se realizó la evaluación antropométrica y encuesta a 1296 alumnos de 70 grados de 22 escuelas y a 362 se les realizó extracción de sangre (Figura 1).

En la Tabla 1, se describen las características de la población.

TABLA 1. Descripción de la población, $n=1296$

\begin{tabular}{|c|c|c|c|c|c|c|c|}
\hline Variable & Frecuencia (\%) & $\begin{array}{c}\text { Porcentaje } \\
\text { de sobrepeso }\end{array}$ & $p$ & $\begin{array}{c}\text { Porcentaje } \\
\text { de obesidad }\end{array}$ & $p$ & $\begin{array}{c}\text { Porcentaje } \\
\text { de exceso de peso } \\
\text { (sobrepeso + obesidad) }\end{array}$ & ) \\
\hline $\begin{array}{l}\text { Grupo de edad } \\
\text { De } 6 \text { a } 8 \text { años } \\
\text { De } 9 \text { a } 14 \text { años }\end{array}$ & $\begin{array}{l}387(29,8) \\
909(70,1)\end{array}$ & $\begin{array}{c}23 \\
25,2\end{array}$ & 0,4 & $\begin{array}{l}18,4 \\
18,5\end{array}$ & 0,9 & $\begin{array}{l}41,3 \\
43,7\end{array}$ & 0,4 \\
\hline $\begin{array}{l}\text { Tipo de escuela } \\
\text { Pública } \\
\text { Privada }\end{array}$ & $\begin{array}{c}818(63,1) \\
478(35)\end{array}$ & $\begin{array}{l}23,6 \\
26,2\end{array}$ & 0,3 & $\begin{array}{l}19,9 \\
15,9\end{array}$ & 0,07 & $\begin{array}{l}43,5 \\
42,1\end{array}$ & 0,6 \\
\hline $\begin{array}{l}\text { Sexo } \\
\text { Masculino } \\
\text { Femenino }\end{array}$ & $\begin{array}{l}627(48,4) \\
669(51,6)\end{array}$ & $\begin{array}{l}23 \\
26\end{array}$ & 0,2 & $\begin{array}{l}22,3 \\
14,8\end{array}$ & 0,0004 & $\begin{array}{l}45,3 \\
40,8\end{array}$ & 0,1 \\
\hline $\begin{array}{l}\text { Nivel de escolaridad } \\
\text { Primaria } \\
\text { Secundaria }\end{array}$ & $\begin{array}{l}998(77) \\
298(23)\end{array}$ & $\begin{array}{l}25,9 \\
19,8\end{array}$ & 0,03 & $\begin{array}{l}19,6 \\
14,3\end{array}$ & 0,04 & $\begin{array}{l}45,6 \\
34,2\end{array}$ & 0,0005 \\
\hline $\begin{array}{l}\text { Convivencia con }<5 \text { años } \\
\text { No } \\
\text { Sí }\end{array}$ & $\begin{array}{c}1219(95,5) \\
58(4,5)\end{array}$ & $\begin{array}{l}24,9 \\
17,2\end{array}$ & 0,2 & $\begin{array}{l}18,3 \\
24,1\end{array}$ & 0,3 & $\begin{array}{l}43,2 \\
41,4\end{array}$ & 0,8 \\
\hline $\begin{array}{l}\text { Escolaridad de la madre } \\
\text { Hasta secundario incompleto } \\
\text { Secundario completo o más }\end{array}$ & $\begin{array}{l}635(49) \\
661(51)\end{array}$ & $\begin{array}{c}25 \\
24,1\end{array}$ & 0,6 & $\begin{array}{l}19,4 \\
17,6\end{array}$ & 0,4 & $\begin{array}{l}44,4 \\
41,6\end{array}$ & 0,3 \\
\hline $\begin{array}{l}\text { Cobertura médica } \\
\text { O. social/prepaga } \\
\text { Sector público }\end{array}$ & $\begin{array}{l}780(60,8) \\
501(39,1)\end{array}$ & $\begin{array}{c}24,9 \\
24\end{array}$ & 0,2 & $\begin{array}{c}18,5 \\
19\end{array}$ & 0,9 & $\begin{array}{l}43,3 \\
42,9\end{array}$ & 0,5 \\
\hline $\begin{array}{l}\text { Desayuno } \\
\text { Sí } \\
\text { No }\end{array}$ & $\begin{array}{c}1044(81,6) \\
235(18,4)\end{array}$ & $\begin{array}{c}24 \\
26,4\end{array}$ & 0,5 & $\begin{array}{l}17,7 \\
23\end{array}$ & 0,06 & $\begin{array}{l}41,8 \\
49,4\end{array}$ & 0,03 \\
\hline
\end{tabular}




\section{Antropometría}

El 43\% (IC 95\%: 40,3-45,7) de los niños presentó exceso de peso (Figura 2).

La CC estuvo aumentada en el 19,1\% (IC 95\%: 16,91-21,19). La proporción varió según el estado nutricional, que fue $78,2 \%$ (IC 95\%: 71,9-83,6) en obesos, $100 \%$ (IC 95\%: 73,54-100) en obesos graves, 15,8\% (IC 95\%: 12,2-20,2) en sobrepeso y $0,28 \%$ (IC 95\%: 0,08-1) en aquellos con peso adecuado.

En relación con los hábitos alimentarios, el 76,84\% (IC 95\%: 74,5-79,1) realizó las cuatro comidas diarias; el 97,7\% (IC 95\%: 96,8-98,4), dos comidas, y el 0,39\% (IC 95\%: 0,2-0,9), solo 1 comida.

\section{Análisis multivariado}

Se realizaron dos modelos de análisis multivariado para evaluar variables asociadas por presentar: a) exceso de peso (sobrepeso + obesidad) y b) obesidad. Las variables edad, nivel educativo de la madre, habitantes por hogar, cobertura de salud, tipo de escuela o hábito de almorzar y cenar no se asociaron a exceso de peso ni a obesidad.

Desayunar estuvo relacionado con menor riesgo de exceso de peso (OR 0,7; IC 95\%: 0,5-0,9) y de obesidad (OR 0,7; IC 95\%: 0,5-0,9). Concurrir al secundario se asoció a menor prevalencia de exceso de peso (OR 0,45; IC 95\%: 0,3-0,7). El sexo masculino se relacionó con mayor riesgo de obesidad (OR 1,7; IC 95\%: 1,3-2,3).

\section{Laboratorio}

Las características sociodemográficas de la submuestra no mostraron diferencias significativas con la muestra total, por lo que resultó representativa de esta. El porcentaje de niños con obesidad fue 18,5\% (IC 95\%: 14,9-22,8) y de exceso de peso, 41,2\% (IC 95\%: 36,2-46,3).

El 100\% de los niños evaluados presentaron valores normales de zinc y ácido fólico; 2 niños tuvieron déficit de vitamina $\mathrm{A} ; 3$, ferritina baja y 2, prealbúmina baja.

Se observó que 16 niños presentaron anemia (4,4\%; (IC 95\%: 2,7-7,1), 13 varones y 3 mujeres (OR 4,6; IC 95\%: 1,3-16,4; p: 0,01). Diez pertenecían al grupo de 12-14 años y 14 tenían peso adecuado para la edad.

Los resultados de las mediciones bioquímicas para lípidos se describen en la Tabla 2.

TABLA 2. Resultados de determinaciones bioquímicas del perfil lipídico, $n=362$

\begin{tabular}{llccc}
\hline Variable & & Porcentaje & \multicolumn{2}{c}{ IC 95\% } \\
\hline Colesterol & Alto & 3,9 & 2,2 & 6,6 \\
& Limítrofe & 15,8 & 12,2 & 20,0 \\
& Normal & 80,4 & 75,8 & 84,3 \\
LDL & Alto & 9,8 & 7,1 & 13,3 \\
& Normal & 90,2 & 86,7 & 92,9 \\
HDL & Bajo & 8,8 & 6,3 & 12,2 \\
& Normal & 91,2 & 87,8 & 93,7 \\
Triglicéridos & Alto & 21,3 & 17,2 & 25,9 \\
& Bajo & 14,1 & 10,8 & 18,2 \\
& Normal & 64,6 & 59,4 & 69,5 \\
\hline
\end{tabular}

IC: intervalo de confianza; LDL: lipoproteínas de baja densidad; HDL: lipoproteínas de alta densidad.

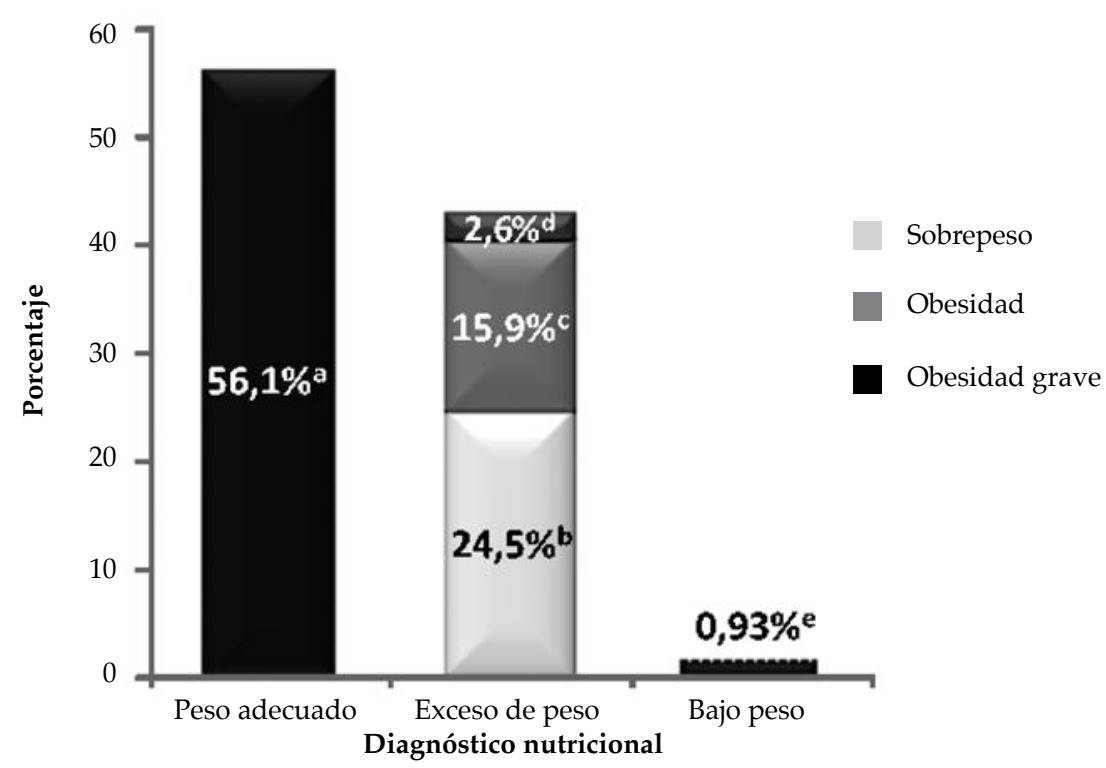

${ }^{\text {a }}$ (IC 95\%: 53,4-58,8); ${ }^{\text {b }}$ (IC 95\%: 22,1-26,8); ${ }^{\text {c }(I C ~ 95 \%: ~ 13,9-17,9) ; ~}{ }^{\text {d }}$ (IC 95\%: 1,7-3,5); ${ }^{\text {e }}$ (IC 95\%: 0,9-3,9) 
El 19,6\% presentó valores de colesterol elevados, que variaron entre un $25,5 \%$ en niños obesos y un $17,5 \%$ en niños con peso adecuado. Esta diferencia no fue significativa.

Un 21,3\% tuvieron triglicéridos altos. Este porcentaje varió al 30,2\% (IC 95\%: 23,4-30,7) en niños con exceso de peso y al 15,02\% (IC 95\%: 10,2-20,1) en aquellos con peso adecuado (OR 2,4; IC 95\%: 1,5-4,1; p: <0,001).

Entre los niños con exceso de peso, los obesos presentaron prevalencias mayores de triglicéridos altos (sobrepeso: 21,95\%; obesos: $40 \%$; obesidad grave: $41,67 \%$ ), y cuando se comparó a los niños no obesos con los obesos, estos últimos tuvieron más del triple de riesgo de presentar triglicéridos elevados que los no obesos (OR 3,3; IC 95\%: 1,86-5,9; p: <0,001).

\section{Ingesta}

La mediana de la energía consumida fue de $2136 \mathrm{kcal}$. La mediana del porcentaje de proteínas respecto del VCT fue del 13,2\%; para hidratos de carbono, del 51,6\% y, para lípidos, del 35,5\% (Tabla 3). La mediana del porcentaje de lípidos respecto del VCT fue mayor que la recomendación $(<10 \%)$, como así también la ingesta de grasas saturadas, mientras que el colesterol dietario y la ingesta de fibra fue menor que lo recomendado. La ingesta de grasas trans representó, aproximadamente, el 2,69\% del VCT, superior a la recomendación $(1 \%)$.

\section{Georreferenciación}

La distribución de los niños fue homogénea en todo el PGP tanto en parámetros bioquímicos como antropométricos. No se encontraron zonas predominantes en ninguna de las variables estudiadas (ver Anexo 3).

\section{DISCUSIÓN}

Los resultados de nuestro estudio mostraron una elevada prevalencia de niños con exceso de peso, mayor que la de otros estudios nacionales. ${ }^{8,9,10}$ Estos resultados son esperables, ya que reflejan la tendencia creciente del sobrepeso y la obesidad y la magnitud de la problemática en el grupo etario seleccionado.

En el informe técnico de la OMS “Dieta, nutrición y prevención de enfermedades crónicas: informe de una consulta mixta de expertos OMS/FAO", se presenta esta temática como un problema de salud pública de cada vez mayor prevalencia, lo que requiere de una estrategia integral para su abordaje. Por ello, se recomienda para prevenir la obesidad en niños y adolescentes un estilo de vida activo, el fomento del consumo

TABLA 3. Ingesta calórica según sexo y edad. Comparación con la ingesta dietaria de referencia*

\begin{tabular}{|c|c|c|c|c|c|c|c|c|c|c|c|c|}
\hline \multirow{4}{*}{ Nutriente } & \multicolumn{6}{|c|}{ De 6 a 8 años } & \multicolumn{6}{|c|}{ De 9 a 14 años } \\
\hline & \multicolumn{3}{|c|}{ Mujeres } & \multicolumn{3}{|c|}{ Varones } & \multicolumn{3}{|c|}{ Mujeres } & \multicolumn{3}{|c|}{ Varones } \\
\hline & \multirow[t]{2}{*}{ IDR } & \multicolumn{2}{|c|}{ Percentil } & \multirow[t]{2}{*}{ IDR } & \multicolumn{2}{|c|}{ Percentil } & \multirow[t]{2}{*}{ IDR } & \multicolumn{2}{|c|}{ Percentil } & \multirow[t]{2}{*}{ IDR } & \multicolumn{2}{|c|}{ Percentil } \\
\hline & & 50 & $25-75$ & & 50 & $25-75$ & & 50 & $25-75$ & & 50 & $25-75$ \\
\hline Energía 24 h calorías / día (kcal) & 1519 & 2024 & $1722-2315$ & 1614 & 2075 & $1548-2404$ & 1856 & 1945 & $1844-2790$ & 2082 & 2394 & 1582-2532 \\
\hline Proteínas (g/día) & $38-114$ & 69,9 & $50-80$ & $40-212$ & 72,5 & 46-92 & $46-139$ & 65,4 & 50-80 & $52-156$ & 75,6 & 58-103 \\
\hline Hidratos de carbono (g/ día) & $171-247$ & 253,9 & $202-323$ & $182-262$ & 239,1 & 198-303 & 209-302 & 254 & $204-329$ & $234-338$ & 307 & $229-383$ \\
\hline Lípidos (g/día) & $42-59$ & 82,5 & $47-104$ & $45-63$ & 86,7 & $50-106$ & $52-72$ & 79,4 & $54-109$ & $58-81$ & 87,2 & $63-126$ \\
\hline Ác. g. saturados (g/día) & * & 25,6 & $16-38$ & * & 30,2 & $20-41$ & * & 24,9 & $16-35$ & * & 29,6 & $19-44$ \\
\hline Ác. g. poliinsaturados (g/día) & $9-19$ & 22,3 & $12-31$ & $10-20$ & 17,6 & $10-27$ & $12-23$ & 20,3 & $12-31$ & $13-26$ & 20,1 & $12-33$ \\
\hline Ác. grasos monoinsaturados (g/día) & --- & 34,4 & $18-34$ & ----- & 30,8 & $18-37$ & ---- & 26,2 & $18-36$ & & 29,6 & $21-42$ \\
\hline Colesterol (mg/día) & * & 233,3 & $145-385$ & * & 252,9 & $133-397$ & * & 249,9 & $146-378$ & * & 292,7 & $190-432$ \\
\hline Fibras (g/día) & 25 & 8,7 & $5,4-11,9$ & 25 & 9,6 & 6-11 & 26 & 8,7 & $6-12$ & $31-38$ & 10,6 & 8-14 \\
\hline Calcio (mg / día) & 800 & 690,6 & $468-1006$ & 800 & 929,4 & $543-1231$ & 1100 & 647,7 & $407-1034$ & 1100 & 775,5 & $320-1115$ \\
\hline Vitamina A (ug/día) & 275 & 478,6 & $291-801$ & 275 & 564,1 & $332-897$ & 420 & 411 & $246-712$ & 445 & 502,2 & $281-768$ \\
\hline Hierro (mg/ día) & 4,1 & 12 & $9,5-17$ & 4,1 & 13,3 & $9-17$ & 5,7 & 12,7 & $10-16$ & 5,9 & 15,3 & $12-21$ \\
\hline Zinc (mg/día) & 4 & 9,8 & $6,5-13$ & 4 & 9,9 & $7-14$ & 7 & 9,2 & $7-12$ & 7 & 11,1 & $8-14$ \\
\hline Folatos (ug/día) & 160 & 496,5 & $343-626$ & 160 & 469,6 & $302-626$ & 250 & 509,5 & 299-706 & 250 & 631 & $381-857$ \\
\hline
\end{tabular}

* As low as possible while consuming a nutritionally adequate diet. (Tan bajo como sea posible, siempre y cuando consuma una dieta nutricionalmente adecuada).

** Ingesta dietaria de referencia Food and Nutrition Board. Institute of Medicine. National Academy of Sciences 2002-2005.

RI: rango intercuartílico; IDR: ingesta dietaria de referencia. 
de frutas y verduras, la reducción de las horas frente al televisor y la restricción del consumo de bebidas azucaradas y de sal. ${ }^{21}$

De las variables socioeconómicas incluidas en los modelos multivariables, el sexo masculino resultó la única asociada a mayor riesgo de obesidad. Esta asociación está descrita en la literatura. ${ }^{22}$

Además, el hábito de desayunar (al menos, 4 veces por semana) resultó ser un factor protector tanto para exceso de peso como para obesidad; esto fue demostrado en distintos estudios. . $3,24^{2}$ En una revisión sistemática, $\mathrm{H}$. Szajewska y col. describieron que aquellos niños que no desayunaban presentaban mayor prevalencia de sobrepeso y obesidad. Desayunar lleva a hábitos alimentarios regulares, ingesta energética adecuada y de alimentos más saludables. Se ha demostrado que quienes no desayunan tienen tendencia a consumir a media mañana alimentos con alto contenido en azúcares y / o grasas. ${ }^{25-27}$

También, independientemente de las variables mencionadas, concurrir a la secundaria se asoció con menos riesgo de presentar exceso de peso. Esto podría deberse a los hábitos alimentarios y a la práctica de actividad física en los escolares de secundaria. ${ }^{28,29}$ No se ha encontrado bibliografía que asocie el nivel de escolaridad con el estado nutricional; se deberán desarrollar nuevos estudios para confirmar esta asociación y evaluar las posibles causas. En este sentido, en relación con la ingesta, se observó que los niños de 6 a 8 años registraban una ingesta superior a la IDR y se notó el exceso en hidratos de carbono y grasas. Esto, vinculado a la mayor prevalencia de sobrepeso y obesidad relacionada con la escuela primaria, permitiría pensar en la influencia del entorno obesogénico en dicho nivel.

No se encontraron diferencias en prevalencia de exceso de peso $u$ obesidad en relación con edad, sexo, tipo de escuela (publica/privada), escolaridad de la madre o cobertura de salud. Tampoco se halló relación con otros factores del ambiente frecuentemente asociados a déficits en el estado nutricional infantil, como edad de la madre o hermanos menores de 5 años. ${ }^{6}$

Un porcentaje importante de niños, principalmente, obesos graves, presentó CC > P 90. El aumento de grasa abdominal está descrito como factor de riesgo para enfermedad cardiovascular (ECV). El estudio Bogalusa Heart Study, en niños de 5 a 17 años, mostró asociación entre la distribución central de grasa determinada por CC, con concentraciones anormales de triglicéridos, lipoproteínas de baja densidad (low density lipoprotein; LDL, por sus siglas en inglés), lipoproteínas de alta densidad (high density lipoprotein; HDL, por sus siglas en inglés) e insulina. ${ }^{30}$ Hirschler y col. describieron, en estudiantes argentinos de 6 a 13 años, una prevalencia de CC > P 90 del 0\% (peso adecuado), $28,6 \%$ (sobrepeso) y $87,5 \%$ (obesidad), similar a nuestros resultados, con el $51 \%$ de los niños con CC > P 90 que presentaban, al menos, un factor de riesgo para ECV. ${ }^{31}$

Del análisis de los datos de laboratorio, se destaca la proporción importante de niños con niveles elevados de colesterol, incluso en niños con peso normal. Algunos estudios refieren que el exceso de peso se asocia con mayor colesterol total, LDL y triglicéridos (TG), a la vez que menor HDL. ${ }^{32}$

De acuerdo con nuestros hallazgos, efectuar dosaje de TG en niños obesos permitiría detectar, aproximadamente, el $40 \%$ de quienes tienen este parámetro aumentado. No obstante, ello deja de lado a los niños con peso adecuado, quienes tienen, probablemente, la mayor representación del componente genético y pueden tener riesgo cardiovascular futuro.

Casi no se detectó déficit de vitaminas y minerales.

La prevalencia de anemia encontrada fue muy baja. Esto es esperable para el grupo etario incluido en el estudio. Se ha descrito una menor prevalencia de anemia en niños escolares, respecto de la primera infancia y la adolescencia, ${ }^{8}$ dada la mayor adecuación entre ingesta y requerimientos nutricionales. La fortificación de harinas según la Ley N. 25630 (2002) puede haber contribuido a la disminución de la anemia en nuestro país. No obstante, se requieren estudios específicos que permitan explicar este hallazgo.

La distribución geográfica de los niños con exceso de peso mostró homogeneidad en todo el PGP. Esto demuestra que esta problemática de salud no es exclusiva de ningún sector ni zonas geográficas en particular.

Para el análisis de la ingesta alimentaria, se utilizó un "recordatorio de 24 h". Esta herramienta presenta como desventaja el subregistro. No obstante, es el instrumento recomendado para estudios poblacionales. Por esto, se complementó con cartillas de alimentos, modelos visuales y formulario de recordatorio estandarizado con alimentos generalmente omitidos. Dada la monotonía de las comidas consumidas, en el programa SARA, se pudo 
analizar la totalidad de los alimentos.

La ingesta de calcio fue insuficiente comparada con la IDR, especialmente, en el grupo de 9 a 14 años, lo que puede explicarse por el aumento en los requerimientos y una inadecuada ingesta de lácteos. ${ }^{33}$

La ingesta de fibras fue baja en todos los grupos. El consumo de grasas totales fue alto a expensas de grasas saturadas; la ingesta de grasas trans duplicó los valores recomendados y el consumo de colesterol fue bajo respecto a la recomendación.

Los niveles elevados de colesterol y triglicéridos en sangre concuerdan con la ingesta excesiva de grasas totales, trans y saturadas observadas, situación que puede aumentar el riesgo de ECV. ${ }^{21,34}$

Este estudio se ha realizado con metodología confiable en las mediciones de peso, talla, cintura y parámetros bioquímicos. Dentro de las limitaciones, puede describirse la falta de datos en lo que respecta a variables como actividad física, tiempo de pantalla, entre otros factores vinculados al exceso de peso.

\section{CONCLUSIÓN}

La prevalencia de exceso de peso fue mayor que lo reportado previamente. El riesgo de obesidad fue mayor en varones y el desayuno surgió como factor protector tanto para sobrepeso como para obesidad. Se reportaron, a su vez, ingesta baja de fibras y elevada de grasas junto con niveles altos de colesterol y triglicéridos en sangre, lo que, en conjunto, refleja que la malnutrición por exceso es un problema de salud pública prevalente.

\section{Agradecimientos}

Al Dr. Hugo Casarsa, Director del HIEMI "V. Tetamanti", por hacer posible la realización del estudio.

A las autoridades de Salud y de Educación del Municipio de General Pueyrredón y de la PBA.

A los Servicios de Gestión y Docencia en Enfermería, Pediatría, Laboratorio, Servicio Social, a la Sala de Soporte Nutricional, al Área de Alimentación y Dietoterapia y a la Jefatura de Consultorios Externos del HIEMI "V. Tetamanti", por proporcionar sus saberes técnicos en la Consulta Interna del Diseño Conceptual.

A las coordinadoras y docentes de la Tecnicatura en Enfermería del "Programa Eva Perón" del Ministerio de Salud de la Provincia de Buenos Aires por facilitar el recurso humano.
A los padres y a los alumnos que participaron desinteresadamente.

A los colaboradores que aportaron sus conocimientos y experiencia para el desarrollo del estudio (ver Anexo 4).

\section{REFERENCIAS}

1. Sánchez Echenique M. Aspectos epidemiológicos de la obesidad infantil. Rev Pediatr Aten Primaria 2012;14(Supl 21):9-14.

2. World Health Organization. Global Strategy on Diet, Physical Activity and Health. Geneva: WHO, 2004. [Consulta: 19 de mayo de 2016]. Disponible en: http:/ / www.who.int/dietphysicalactivity/goals/en/index.html

3. Ballesteros Arribas JM, Dal-Re Saavedra M, Pérez-Farinós $\mathrm{N}$, et al. La estrategia para la nutrición, actividad física y prevención de la obesidad (estrategia NAOS). Rev Esp Salud Pública 2007;81(5):443-9.

4. Aranceta Bartrina J, Pérez Rodrigo C, Ribas Barba L, et al. Epidemiología y factores determinantes de la obesidad infantil y juvenil en España. Rev Pediatr Aten Primaria 2005;7(Supl 1):S13-20.

5. Grandy G, Weisstaub G, López de Romaña D. Deficiencia de hierro y zinc en niños. Rev Bol Ped 2010;49(1):25-31.

6. Calvo EB, Aguirre P. Crisis de la seguridad alimentaria en la Argentina y estado nutricional en una población vulnerable. Arch Argent Pediatr 2005;103(1):77-90.

7. Rivera JA, Barquera S, González-Cossio T, et al. Nutrition transition in Mexico and in other Latin American countries. Nutr Rev 2004;62(7 Pt 2):S149-57.

8. Encuesta Nacional de Nutrición y Salud, ENNyS. Documento de Resultados, 2007. Buenos Aires: Ministerio de Salud. [Consulta: septiembre de 2012]. Disponible en: http: / / www.msal.gob.ar/images / stories / bes / graficos / 0000000257cnt-a08-ennys-documento-deresultados-2007.pdf.

9. Ferrante D, Linetzky B, Ponce M, et al. Prevalencia de sobrepeso, obesidad, actividad física y tabaquismo en adolescentes argentinos: Encuestas Mundiales de Salud Escolar y de Tabaco en Jóvenes, 2007-2012. Arch Argent Pediatr 2014;112(6):496-503.

10. Kovalskys I, Indart Rougier P, Amigo MP, et al. Ingesta alimentaria y evaluación antropométrica en niños escolares de Buenos Aires. Arch Argent Pediatr 2013;111(1):9-14.

11. Duelo Marcos M, Escribano Ceruelo E, Muñoz Velasco F. Obesidad. Rev Pediatr Aten Primaria 2009;11(Supl 16):239-257.

12. Censo Nacional de Población, Hogares y Viviendas 2010. Instituto Nacional de Estadísticas y Censos. Argentina. [Consulta: septiembre de 2012]. Disponible en: http:// www.indec.gob.ar/.

13. Benoist B, McLean E, Egli I, et al. Worldwide prevalence of anaemia 1993-2005. Base de datos mundial sobre la anemia delaOMS, Ginebra, Organización MundialdelaSalud, 2008. [Consulta:23 de agostode 2017]. Disponibleen: http: / / apps. who.int/iris/ bitstream/10665/43894/1/9789241596657_ eng.pdf.

14. Comité Nacional de Crecimiento y Desarrollo. Sociedad Argentina de Pediatría. Guía para la evaluación del crecimiento físico. 2013. [Consulta: marzo de 2013]. Disponible en: http://www.sap.org.ar/docs/ publicaciones/libro_verde_sap_2013.pdf.

15. Comité Nacional de Nutrición. Guías de práctica clínica para la prevención, el diagnóstico y el tratamiento de la obesidad. Arch Argent Pediatr 2011;109(3):198-203.

16. Gil Hernández A. Métodos para la evaluación de la ingesta de alimentos. En Tratado de Nutrición. Composición y calidad Nutritiva de los Alimentos. 2. ${ }^{\text {da }}$ ed. Madrid: Panamericana; 2010:594-6.

17. López BL, Longo NE, Carballido PM, et al. Validación del uso de modelos fotográficos para cuantificar el tamaño de las porciones de alimentos. Rev Chil Nutr 2006;33(3):480-7.

18. Patrones Internacionales de Crecimiento Infantil delaOMS. Buenos Aires: Ministerio deSalud. [Consulta: septiembre de 
2012]. Disponible en: http:/ / www.ms.gba.gov.ar/sitios / maternoinfantil / files/2012/05/1-evaluacion_curvas_ final1.pdf.

19. Torresani ME. Manual Práctico de Dietoterapia del Niño. $1^{\mathrm{a}}$ Cátedra de Dietoterapia del Niño. Carrera de Nutrición de la Universidad de Buenos Aires. Buenos Aires: Akadia; 2011.

20. Dietary Reference Intakes (DRIs): Estimated Average Requeriments. [Consulta: agosto de 2017]. Disponible en: https: / / www.nap.edu/read/13050/ chapter/24\#1105.

21. Organización Mundial de la Salud. Dieta, Nutrición y Prevención de Enfermedades Crónicas: informe de una Consulta Mixta de Expertos OMS/FAO. Ginebra: OMS, 2003. Serie de Documentos Técnicos: 916. [Consulta: 12 de marzo de 2014]. Disponible en: www.who.int/nutrition/ publications / obesity/WHO_TRS_916_spa.pdf.

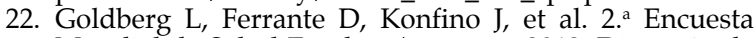
Mundial de Salud Escolar. Argentina 2012. Dirección de Promoción de la Salud y Control de Enfermedades No Transmisibles. Ministerio de Salud. Noviembre de 2013. [Consulta: febrero de 2014]. Disponible en: http:/ / www. msal.gob.ar/ent/images/stories/vigilancia/pdf/201409_informe-EMSE-2012.pdf.

23. Berta E, Fugas V, Walz F, et al. Estado nutricional de escolares y su relación con el hábito y calidad del desayuno. Rev Chil Nutr 2015;42(1):45-52.

24. Henríquez Sánchez P, Doreste Alonso J, Laínez SevillanoP, et al. Prevalencia de obesidad y sobrepeso en adolescentes canarios: relación con el desayuno y la actividad física. Med Clin 2008;130(16):606-10.

25. Nicklas TA1, Reger C, Myers L, etal. Breakfastconsumption with and without vitamin mineral supplement use favorably impacts daily nutrient intake of ninth-grade students. I Adolesc Health 2000;27(5):314-21.

26. Wyatt HR, Grunwald GK, Mosca CL, et al. Long-term weight loss and breakfast in subjects in the National Weight
Control Registry. Obes Res 2002;10(2):78-82.

27. Szajewska H, Ruszczynski M. Systematic review demonstrating that breakfast consumption influences body weight outcomes in children and adolescents in Europe. Crit Rev Food Sci Nutr 2010;50(2):113-9.

28. Arriscado Alsina D, Muros Molina JJ, Zabala Díaz M, et al. Influencia del sexo y el tipo de escuela sobre los índices de sobrepeso y obesidad. Rev Pediatr Aten Primaria 2014;16(64):e139-46.

29. Poletti HO, Barrios L. Sobrepeso, obesidad, hábitos alimentarios, actividad física y uso del tiempo libre en escolares de Corrientes (Argentina). Rev Cubana Pediatr 2007;79(1). [Consulta: 14 de octubre de 2016]. Disponible en:http:/ / scielo.sld.cu/ scielo.php?script=sci_ arttext\&pid=S0034-75312007000100006\&lng=es

30. Freedman DS, Dietz WH, Srinivasan SR, et al. The relation of overweight to cardiovascular risk factors among children and adolescents: the Bogalusa Heart Study. Pediatrics 1999;103(6 Pt 1):1175-82.

31. HirschlerV,DelfinoAM,ClementeG,etal. ¿Eslacircunferencia de cintura un componente del síndrome metabólico en la infancia? Arch Argent Pediatr 2005;103(1):7-13.

32. Barja $S$, Barrios $X$, Arnaiz $P$, et al. Niveles de lípidos sanguíneos en escolares chilenos de 10 a 14 años de edad. Nutr Hosp 2013;28(3):719-25.

33. Zapata ME, Rovirosa A, Carmuega E. Estudio de patrones de consumo de lácteos y calcio en población argentina CESNI 2012. [Consulta: 13 de octubre de 2016]. Disponible en: http: / / www.cesni.org.ar/archivos/observatorio/ PAPGCL.pdf.

34. Ballesteros-Vásquez M, Valenzuela-Calvillo L, ArtalejoOchoa E, et al. Ácidos grasos trans: un análisis del efecto de su consumo en la salud humana, regulación del contenido en alimentos y alternativas para disminuirlos. Nutr Hosp 2012;27(1):54-64. 


\section{ANEXO 1}

Planilla de recolección de datos socioeconómicos

Esta encuesta forma parte del estudio para conocer el estado nutricional de los niños en edad escolar del Partido de Gral. Pueyrredón. Recuerde que los datos no serán usados para otros fines ni se darán a conocer los nombres y datos de los niños en los informes. Le pedimos que por favor se tome $\mathbf{1 0}$ minutos para completarla, ¡muchas gracias!

FECHA DE ENCUESTA
Apellido y nombre del niño/a:

DNI:

Fecha de nacimiento: Sexo: Masculino Femenino

Lugar de nacimiento:

Domicilio:

Escuela: Grado y División:

1. ¿Qué comidas realiza el niño/niña habitualmente? (puede marcar más de una opción) por lo menos 4 veces por semana

Desayuno

Almuerzo

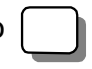

Merienda

Cena

2. ¿Cuál es la edad de la madre o cuidador? Edad: años.

3. Nivel de instrucción de la madre o cuidador: (marque solo el último nivel alcanzado). Ninguna Primario incompleto Primario completo Secundario incompleto Secundario completo

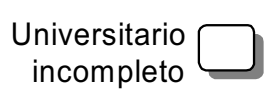
Universitario completo

4. Cobertura en salud. ¿Qué tipo/s de cobertura médica o seguro de salud tiene el niño en la actualidad?

Obra social / Medicina prepaga

Sin cobertura, se atiende en la Salita u Hospital

5. ¿Quiénes viven en tu hogar, incluyéndote a vos?

\begin{tabular}{|l|l|}
\hline $\begin{array}{l}\text { Anote el parentesco con el niño } \\
\text { (Ej.: padre o esposo de la madre, madre, hermano, tío, etc.) }\end{array}$ & EDAD \\
\hline & \\
\hline & \\
\hline & \\
\hline & \\
\hline & \\
\hline & \\
\hline
\end{tabular}


ANEXO 2

Evaluación del estado nutricional antropométrico, bioquímico y de ingesta alimentaria, de niños entre 6 a 14 años, General Pueyrredón, Buenos Aires, Argentina

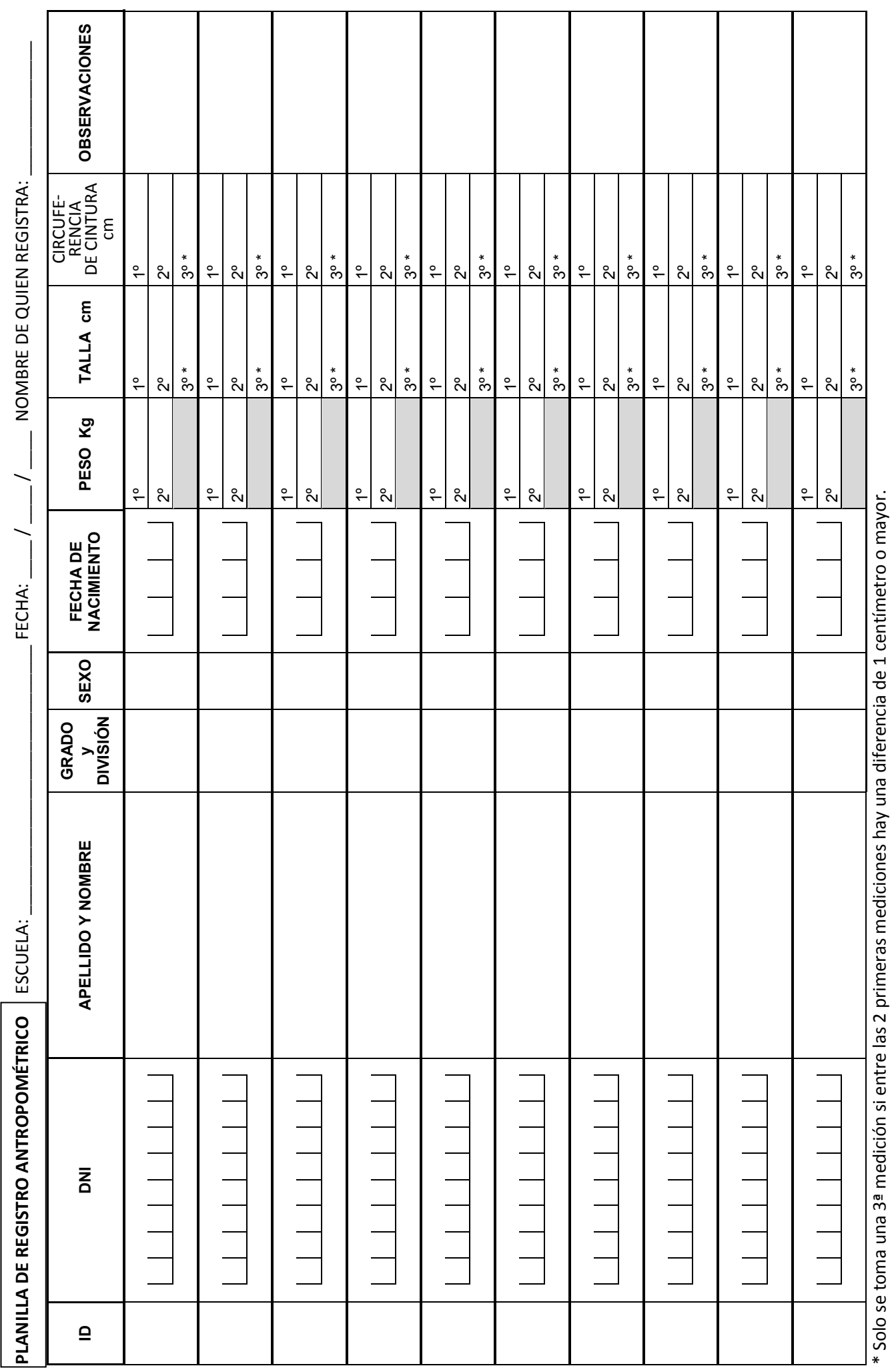




\section{ANEXO 3}

Distribución de niños según el estado nutricional.

\section{Partido de General Pueyrredón}

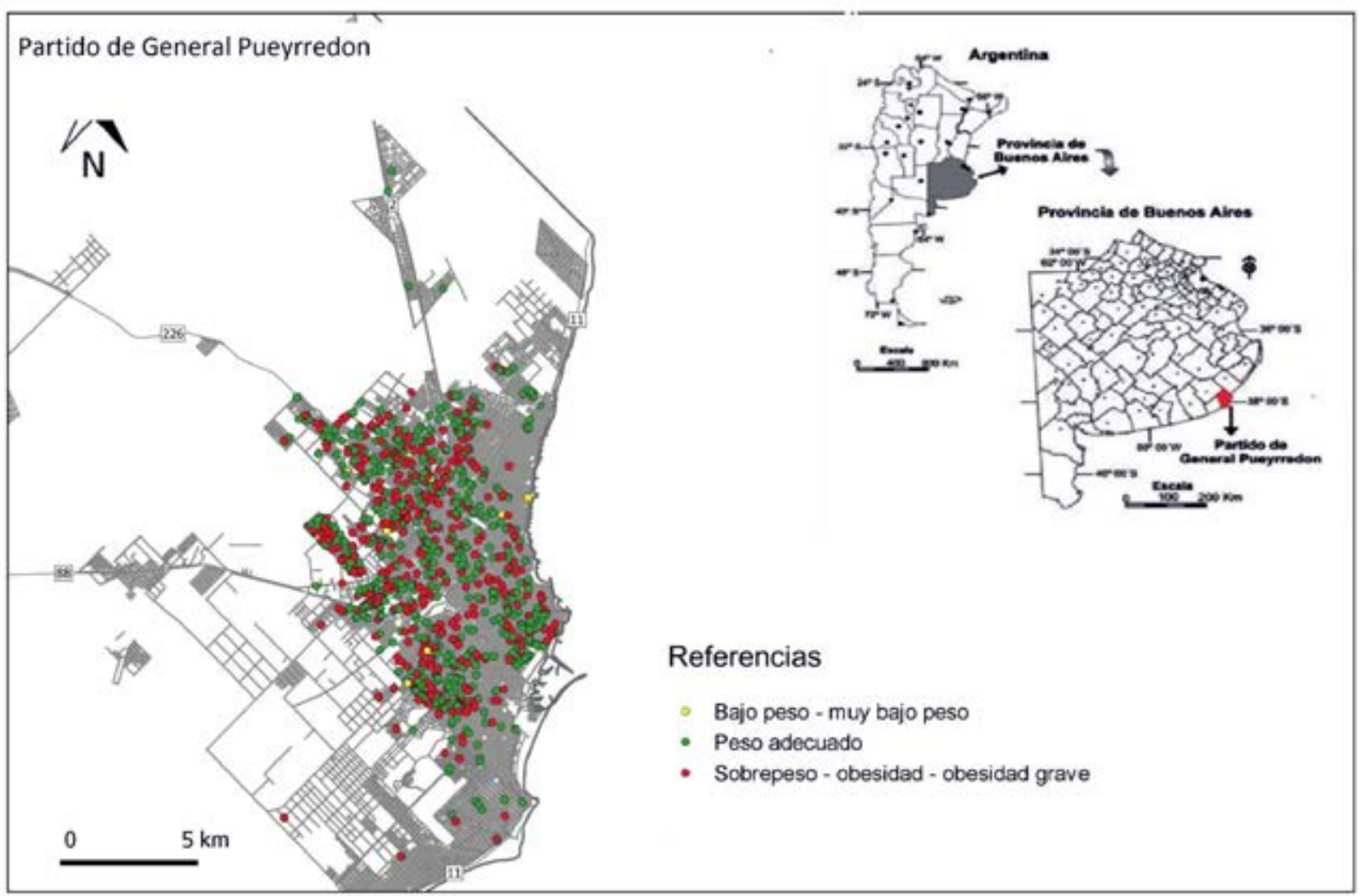


e46 / Arch Argent Pediatr 2018;116(1):e34-e46 / Artículo original

\section{ANEXO 4 \\ Colaboradores}

Hospital Interzonal Especializado Materno Infantil “V. Tetamanti”: Lic. Silvana Pinto, Nutr. Claudia Guridi, Lic. Carolina Echarri, Lic. Natalia García, Lic. Fernanda Paglione, Lic. Rocío Silva, Lic. Agustina Basso, Lic. Paola Buscaglia, Lic. Silvana Barragán, Lic. Malena Bouza,

Dra. Pilar López, Dra. Ana Cabral, Dra. María Rossi, Lic. Cruz Costantini, Lic. Eugenia Epele,

Lic. Adriana Mascolo, Lic. Daniela Ortega, Lic. Estefania Agostini, Lic. Estefanía André, Lic. Mercedes Pereira, Enf. Marta Rus, Enf. Sonia Martinez, Enf. Silvina Pose, Enf. Nieves Alanis, Aux. Enf. Carmen Monroy, Aux. Enf. Cristina Gómez, Enf. Almirón María, Enf. Fabián Vázquez, Enf. Laura Armúa, Dra. Nilda Parisi, Dra. Alina Guarino, Lic. Ana Algañaraz, Lic. Patricia Pastrana, Lic. Estela Todisco, Lic. Delia Fagoaga, Bioq. Paola Rolandi, Bioq. Valeria Cavallaro, Bioq. Magali Ramadori, Bioq. Carolina Noya, Téc. Marchena Flavia, Promotora Elvira Cisneros, Promotora Sandra Mascioli, Promotora Juliana Girard Riat, Promotor Sergio Lombardo.

Secretaría de Salud del Municipio de General Pueyrredón: Lic. Soledad Iacoponi, Dra. Iabranka Juhsic, Dr. Edgardo Marambio Catan, Lic. Estefanía Oteiza, Lic. Constanza Pagano, Lic. María Teresa Martínez.

Secretaría de Educación del Municipio de General Pueyrredón: Dr. Martín Zemel.

Universidad Nacional de Mar del Plata: MSC Mónica Tomas, Cart. Virginia Bernasconi, Cart. Marcelo Farenga.

Jefatura de Región 19 de Educación de Gestión Privada. Dirección Provincial de Gestión Privada: Lic. Ana Carolina Di Alessio.

Universidad Nacional de La Plata: Dr. Marcelo Tavella.

Instituto de Desarrollo e Investigaciones Pediátricas (IDIP) "Prof. Dr. Fernando E. Viteri": Dr. Horacio González, Bioq. Natalia Matamoros, Bioq. Fernanda Santandreu, Bioq. Enrique Martins, Bioq. Ana Varea.

Ministerio de Salud de la Provincia de Buenos Aires: Lic. Nadia Attie, Lic. Paula Das Neves, Lic. Mónica López.

Universidad Nacional de Buenos Aires: Lic. Natalia Elorriaga.

Florida International University (EE. UU.): Dr. Nöel Barengo.

Instituto de Salud Pública de México: Dr. Juan Rivera.

Instituto de Nutrición y Tecnología de Alimentos de la Universidad de Chile (INTA): Dr. Ricardo Uauy. 